\title{
PUBLIC RELATIONS AND JOURNALISM
}

The paper offers the findings of possible interconnection between journalism and public relations. It was created as a reaction to the need to explore the level of influence (and impact) of public relations worker on journalism. This need was accompanied by the attempt to look into the issue both from the aspects of journalist and public relations worker. We focused on the exploration of media environment (in relation to media market) with respect to possibilities of being influenced by marketing, specifically by the area of public relations.

Keywords: public relations, journalism, interconnection

\section{Introduction}

The issue of media environment is very interesting, but too complicated to be mapped and analyzed in its absolute entirety. Nowadays, the questions dealing with the penetrating of the infotainment into the area of news service are raised, together with the questions of increasing interconnections with some areas of marketing (advertising, public relations).

Regarding the fact that the dynamic marketing progress disturbs the traditional procedures in the area of journalism, it has become the subject matter of wide professional and academic interest, and vice versa, the marketing workers are interested in the attributes of media environment-functioning and creation of media content.

The knowledge and perceptive procedures of the functioning of media and marketing environment are the precondition to the determination of the level of the usage of marketing (or PR) contents in seemingly journalistic manifestations.

The article presents particular results of the research which has been motivated by the need to explore the level of influence (and impact) of public relations worker on journalism. This need was accompanied by the attempt to look into the issue both from the aspects of journalist and public relations worker. We focused on the exploration of media environment (in relation to media market) with respect to possibilities of being influenced by marketing, specifically by the area of public relations.

\section{Research}

The aim of the research was to map mutual similarities, differences and interlinks between both areas (journalism and public relations) and to set the level and causes of the penetrating of information from press releases into the area of journalism.
An important inspiration for the research was one of the journalistic rules that a journalist should use information from different sources at his/her work and the feeling that this rule is often forgotten and overlooked and PR materials have become an essential source medium for journalism.

To reach the aim we set five elementary hypotheses and to increase the relevance of the research we used three research methods. The results of the research could be consequently compared and interpreted in wider connection. The basic pillar of the research was the content analyses of texts (press releases and selected media contents). We used also questionnaires and non-structured qualitative interviews with PR workers to ensure the complexity of information.

Press releases and their reflection (media contents in the form of editorial contributions) in the segment of print media (yellow and serious journalism) and their online versions on the Internet, as well as the editorial contributions of press agencies are the subject matter of our research. The further stage of the research was based on questionnaires and interviews with PR workers and journalists.

We focused on the exploration of media environment stressing the borderline connections with marketing, primarily with the area of public relations. The aim included several points:

- To confirm or confute the possibility of the penetrating of information from the press releases to the area of news service.

- To determine the influence of press release on the number of media outputs.

- To characterize the relationship between PR workers and journalists.

- To analyze the use of press release as an information source. [2]

\footnotetext{
* Slavka Pitonakova

Faculty of Humanities, University of Zilina, Slovakia, E-mail: slavka.pitonakova@fpv.uniza.sk
} 
The research realization was conducted in several phases:

The first phase of the research: the study of relevant texts with the aim to understand and analyze up to now knowledge relevant for the research theme.

The second phase of the research: the determination of hypotheses and the proposal of methodology (including data gathering from subjective enunciations).

The third phase of the research: The research realization (content analysis, questionnaire, qualitative interviews).

The fourth phase of the research: The analysis of data, interpretation of findings and evaluation of the research.

The selection of research sample

The selection of research sample reflected the current situation in Slovak media environment. Selected press releases and media contents (technologically quite simple which allowed the realization of their content analysis), the answers of respondents in questionnaires and interviews with PR workers were the subject matter of the research.

Before the research realization five research hypotheses were stated:

Hypothesis No. 1: More than one third of published press releases are the basic information source for the creation of journalistic contributions.

Hypothesis No. 2: Journalists use press releases for their journalistic contributions without adequate verification of information from different sources

Hypothesis No. 3: The theme of press release influences the level of its importance for journalists (public).

Hypothesis No. 4: PR workers economically pressure journalists.

Hypothesis No. 5: Positive relationships between PR workers and journalists are the main reason of the press release penetrating into the area of news service.

\section{Methodology of research}

We used the combination of three methods in the methodology of our research - content analysis, questionnaire and qualitative interview. The reason was an attempt to increase the relevance of the research.

The questionnaire was validated by means of questionnaire for PR workers and content analysis. The answers in questionnaires were compared with the results of analysis of particular media outputs.

We tried to increase the reliability of content analysis by the rigorous determination of percentage exploitability of press releases and percentage of press releases in media outputs. Only the identical (not paraphrased) words, sentences and paragraphs were considered as a relevant unit for the analysis in each case.

The aim of the analysis was to determine the level of equivalence (expressed in the percentage of press releases on media response) with the media outputs in print media. We explored whether the selected press releases in newspapers, magazines and online media are published in:

- identical version,

- partially changed, not appended version,

- partially changed and appended version,

- not published.

We also explored the possible interconnections between the themes of press releases and their attractiveness to journalists (i. e. which of the press releases are the most interesting for the journalists). When choosing the press releases we focused on the following areas: politics, economy, culture and edifying activities.

The content analysis of the products required the specifying of documents for the research. We concentrated on written, publically available institutional documents (print, online media and press releases), which were examined in the context of preset themes without regard to periodicity of their occurrence. We mainly focused on press releases, and then print and online media published in Slovakia. The underlying condition for the subsuming of an article (in printed or electronic form) to the research was its particular or general equivalence with examined press release published on the Internet.

The selection of press releases was based on the method of coincidental selection in respect to the formerly chosen thematic fields and the subject matter of further analysis were only the press releases which were completely or partially transformed into the form of a particular media output (article in printed or electronic media).

30 press releases were the subject matter of our research. 14 articles published in different media with the name of their redactors (redaction, agency) met our requirements for detailed analysis (disclosure of similarity or identity with the prototext of press release).

We considered following as a classification unit:

- integrated contribution specified by the title "press release",

- integrated contribution tagged by a title.

We defined a word as a coding unit and selected following elements for the classification:

- media output: yes (number)/no (x),

- frequency (media response of a press release) - the description of media which published the articles inspired by the particular press release,

- extensity (the percentage of a press release in media output) the prevalence of the code - the number of the words in an article which are identical with the words of the compared press release.

Following elements were evaluated in the analysis of press releases [according to 1 ]:

- title,

- author of the press release,

- publication date, 
- press release focus,

- press release theme,

- number of words,

- media response of press release (frequency): yes (description)/ no $(\mathrm{x})$ the use of press release in media: percentage

We added following elements to the analysis of media outputs:

- media response of an article (in the case if a press release was published by a press agency),

- main source of information: specified (specification)/non-specified,

- other sources of information: yes (description)/no (x),

- the prevalence percentage of a press release in an article (extensity): percentage of the number of identical words with prototext of the press release.

Following criteria and quantification method were chosen for the comparison of the selected press release with particular media output (the article):

Exploring the equivalence of the press releases with the media outputs we focused on the measurement of the number of identical words, sentences and paragraphs, as well as on the frequency of the prevalence of a word (coding unit) in the text.

Regarding the fact that it is necessary to adjudicate the texts in their context, we assumed some controversies in analyses. For the minimizing of different aberrations (specifically subjective evaluation) and the improvement of data quantification we specified that power and intensity of examined phenomenon would be expressed in percentage (percentage of press release in media output and percentage of the press release use.)

After the realization of the content analysis we can state several important findings. In the preparatory phase of the research we assumed that the researches conducted by several professionals in the area would correspond to the outcomes of our analysis. The mapping and analyzing of 30 different press releases allowed us to submit that the editors of print media in news service did not use press releases in any case.
The specific focus was laid on the attempt to find connections between press releases and news service in so called serious daily papers in Slovakia. No direct connection was found. Surprisingly, we have found out that in the case of similarities between press releases and media response the only sources used by the editors of specific media was from the prepaid service of press agencies.

From the total number of examined press releases 18 press releases had no media response. The other 13 press releases were primary (and almost the unique) information source for the news contributions. All articles state as their main information sources press releases or speaker (i. e. a PR worker is an information). In no case other information source was specified.

When analyzing the texts we focused on the facts mostly interesting for press release editors. We found out that the press releases from the area of politics are the most attractive (five from the total number of seven press releases are from the area of politics). The second place is occupied by the press releases focused on the edifying activities and health campaigns (three from five press releases). This fact may be explained from two points of view. First, an ambition of editors to help with the advertising of positive activities, and second, the journalist outcome corresponds to the theory of gatekeeping, which means that journalists prefer the news having so called human touch. The press releases from the area of culture were not as interesting (three from seven press releases) and the press releases from the field of economy were the least interesting [2].

It is worthy to mention the composition aspect of the texts as well. If the editors are inspired only by the press releases they rarely change the texts and usually keep the original composition. The titles are only a transformation of press release title or its main idea. Creativity and original asset of an author are not usual; we may say that it is very rare.

The research followed the aim to explore particular phenomena also from the perspective of a PR worker. From his/her perspective we must conclude that we may consider PR as successful if more than one third of published press releases appear in media in an almost original form.

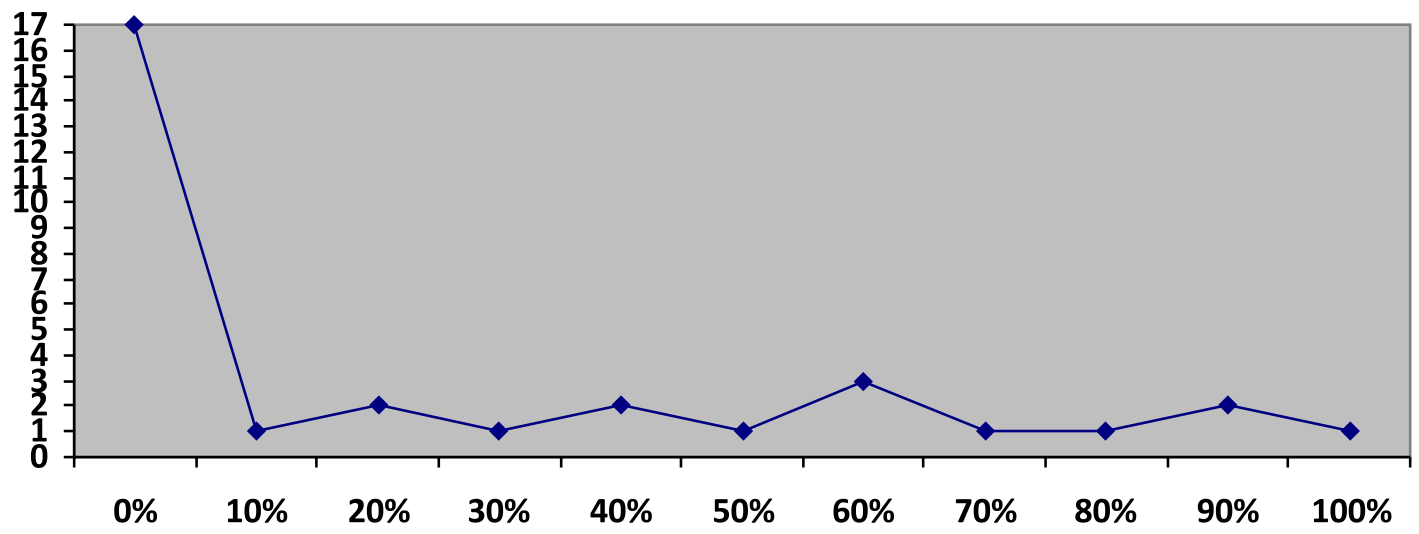

Fig. 1 The share of press releases editorial contributions in relation to the number of articles 
On the basis of content analysis we may state that the hypotheses No. 1, 2 and 3 were proven true. The facts were further verified by means of a questionnaire.

While realizing our research we sent e-mails to 100 journalists and to the same number of PR workers who could answer the questions in questionnaires anonymously by means of independent web application. In accordance with our assumption more PR workers than journalists joined the research (the return of questionnaires was $40 \%$ ). The return of questionnaires from the journalists was only $20 \%$. The similar situation repeated when we tried to realize qualitative interviews with journalists. While all of the three addressed PR workers agreed on the interviews (the interview was conducted by means of phone call or e-mail), no journalist agreed to participate.

By means of particular items in the first part of the questionnaires we explored necessary demographic data. We were interested in the age of the respondents and the time (in years) they had spent in spotted areas (PR, journalism). Other demographic data (gender, ...) were considered in our research as irrelevant.

The questionnaire had 14 items which were divided into four groups as they could correspond to the need to verify preset hypotheses. The groups of questions aimed to map the level of press release publishing in print and online media as well as the verification of information in press releases and other sources, relationships between PR workers and journalists and possible pressure on journalists caused by PR workers. The questionnaire was created in a way to ensure respondents' comfort when answering the questions. We assumed that both target groups have time-consuming and stressful jobs, which means that in the case of a complicated questionnaire they would not have answered at all.

The average age of the PR workers was 35.28 years; the youngest participant was 26 years old, the oldest 59 years old. The average age of the journalists was lower -31.76 years; the youngest participant was only 19 years old and the oldest 59 years old. The results show that in both areas (PR and journalism) mostly young people work. The professional experience of the journalists is less than five years and experience of the PR workers is up to 10 years. The differences are clear mainly in connection with the last indicator (period of work experience is $10-15$ years) which adverts to the smaller number of PR workers. This fact may be explained by the young tradition of PR profession.

31 PR workers from the total number of 40 think that journalists follow internal editorial rules for their work with PR materials. Only five of them are convinced that journalists ignore the rules. Regarding the fact that in our questionnaire we explored how long the respondents had worked in the professional area, we were interested in the answers of the most experienced PR workers. Those who have worked in PR more than 15 years (only two) concurred in the fact that journalist rather respect editorial rules. This finding correlated to the outcomes of our content analysis.

The journalists conveyed the same - 15 journalists from the total number of 20 answered that journalists follow internal edito- rial rules. Surprisingly, 5 journalists could not give clear answer yes/no. More than half of the PR workers (25) are convinced that journalists publish PR materials as editorial contributions. On the contrary, more than half journalists (11 from 21) think that it does not happen. Most journalists (4) who think that their colleagues work this way have been in the area of journalism less than 5 years. 35 from 40 people working in PR confessed that their PR material had been published in its original form, whilst the most positive experience with this have the PR workers who have worked in PR less than 5 years. None of them has had an experience with at least one unpublished PR material or TS. The journalists had slightly different attitude. 18 journalists answered clear "no", only three of them answered "yes". From these three two have worked as journalists less than $10-15$ years, one more than 15 years. These findings enable us to conclude that the higher age of a journalist, the more opportunities (and possibly mistakes as well) may influence him/her at their work.

17 journalists from the total number of 21 answered that they verify information. The answers do not correspond to the results of the content analysis from which resulted that if a journalist uses a TS he/she does not verify it in other sources. This discrepancy may be explained by the fact that there were more print media editors than editors from press agencies involved in our research and their answers increased the number of positive answers.

PR workers are convinced that they do not put pressure on journalists (27), but 12 from 40 confessed that PR agencies intrude media workers. The journalists think differently. 13 journalists think that PR agencies put pressure on them. It is necessary to complete the information that $23 \mathrm{PR}$ workers and 13 journalists think that a present a journalist is offered does not influence the content of media outcome. Simultaneously, a smaller part of PR workers and journalists thinks the opposite.

In the sixth part of the questionnaire both target groups mention different kinds of smaller or bigger presents, which are offered or accepted. From among them the most valuable are notebooks, software applications, electronics, jewelry, holidays, mobile phones, wellness stays, trips abroad, invitations to cultural or sport events, alcohol (luxury wine), beer barrel, food and cosmetics, exclusive pens, ski tickets, 500 EUR, ...

Some of the PR workers and journalist think that they haven't had any experience with presents, but one of them admitted an offer of a trip to Monza F1. Regarding considerable benefits (question No. 10), which might lead to concealing information, the PR workers ( 39 from 40) adduced that they had never been offered any of them and the journalists unanimously answered that they had never accepted any of them.

Hypothesis No. 4 not proven

Regarding the last hypothesis we may conclude that 38 PR workers admit benefits of positive relationships with journalists in the time of critical communication. Concerning the TS publishing as editorial contributions, more PR workers think that the main reason is busyness of journalists, their non-professional attitude to work and the last place is occupied by positive relationships with 
them. Some of the PR workers provided further explanations for publishing their press releases. For example, three PR workers think that the reason is the quality of sources, professional PR and serious information ("well-taken and informative value of PR"). One PR worker said the following about journalists' tendency to subscribe PR: "They are not as good at a certain area, they are too busy to study the topic, there is no investigative journalism in Slovakia”.

A major part of PR workers does not admit their dependence on journalists (30), only 8 of them think that PR workers depend on journalists; two expressed that journalists depend on PR materials (workers).

The journalists think the opposite regarding PR workers and journalists. 10 from 21 respondents answered that the relationship is equivalent, but almost the same number of them think (11) that PR workers depend on journalists. No journalist expressed an opinion that journalists depend on PR workers.

The journalists' view on the publishing of TS as editorial contributions is also interesting. More than half of them (11) mentioned non-professionalism of their colleagues; the second place is taken by busyness of journalists. Positive relationships with PR workers as the main reason for this are stated (the same as in the evaluation of the PR workers) as the least relevant reason. A detailed view on the respondents' answers is also interesting. Three journalists admitted that they had published a PR material as their own contribution. As the reason for this they stated their busyness and positive relationships with PR workers. One of them openly confessed nonprofessional attitude. All of them have been in the area of journalism more than 10 years. One of the respondents commented the situation: "it is a usual thing, that is why it is a PR article - the point is the quality. The problem is not in the content, but in the form", another one stated: "I don't think that journalists sign PR with their own names". The differentness illustrates the controversy of the theme and inconsistence of reactions [2].

\section{Conclusion}

The contribution offers a view on the issue of dynamic development of media environment with regard to possibility to use PR materials as a specific informative tool. The challenge was to write a text providing interdisciplinary perspective on the problem, which has been (because of many reasons) unnoticed.

Accepting the uniqueness of media space we remind ourselves that journalism has been traditionally profiled as an area with high professional ideals, which are not in accordance with practical achievements of journalists. Public, professionals, as well as practitioners from the area of media point out non-standard examples of the activities of journalists and media workers. Together with the poor quality of texts and the lack of investigation, they criticize non-standard relationships with PR people. To advocate journalism one may say that in comparison with PR, journalism is considerably financially undersized. This may lead to different kinds of "cooperation" between journalists and PR workers (sometimes the problem is not the "step over" of journalists toward PR workers, but also back to media environment).

Regarding the theme several researches have been realized showing some models of relationships between journalism and PR. Some of them point out the fact that PR determined journalism and that there is a mutual interconnection between PR and journalism, which has also been proven in our research (mainly by means of the interviews and the last question in questionnaire, in which the respondents openly expressed their opinion on the questionnaire and relationships between PR workers and journalists).

It is necessary to realize the status of both disciplines in media space and to intensify our perception of reality proving the fact that there is no partnership (in its real meaning). The questions such as how often, how much and in what way media use advertising materials and press releases must be relevantly answered. The reason is that PR is a specific area as its results are immeasurable. There are some specialists who document the results by the quantification of the spatium in media (the calculation in squared centimeters), but these numbers may not be (and are not) authoritative, as the spatium of a PR material does not correspond to the publicity reached by it [3] (and finally, PR itself is not just about "media relations").

In our research we have noticed that if journalists use a TS as a source, they do not change its original text. They paraphrase the title, copy particular parts (some of them are slightly amended afterwards) and modify the structure. The equivalence with the prototext is more than $30 \%$.

This finding corresponds to the attempt of PR workers to use the methods of journalists which results in qualitatively high-written and professional texts (attributes of the inverted pyramid, "human touch", story). This is proven by the journalists' opinions from the questionnaire, as well as by the interviews with PR workers.

Press releases are interesting also from their thematic point of view. The influence of a theme on TS selection as an informative source is essential regarding the theory of gatekeeping (with an emphasis on "human touch"). Commonly used press releases are from political environment (specifically those having the character of scandals and suspicions). These are followed by the TS from the area of edifying activities and culture. The least common TS have economic character.

According to PR workers and journalists (self-critically), the most common reasons of the penetrating of PR information into the area of media are busyness and non-professionalism of journalists. By means of the questionnaire and interviews and concerning different theories, we have explored the opinions of practitioners on the relationship between PR and journalism. None of the journalists thinks that journalists depend on PR, 8 PR workers think that journalists are dependent on PR, but both professions usually agree on the fact that their relationship is equal.

Nowadays, it is necessary for both professions to realize that $\mathrm{PR}$ is at the peak of its expansion and it is and will be an important 
informative source for journalism. On the other hand, media space and journalists is a very dynamic organism, full of people with ambitions to work professionally, and therefore there is a high probability that the tries to set the clear rules for the work with PR materials remain a crucial theme of journalists.

Likewise, it will be important to identify limits between PR and journalism, as some of the journalists' answer and PR workers indicate that some practitioners consider PR publishing as standard editorial method.

Even from this point of view our research represents an insight into the media environment. Considering the situation of media, journalism and public relations in Slovakia, as well as the fact that the issue has been worked out only from the perspective of particular disciplines and not interdisciplinary, our research may be even more inspirational. Moreover, some of the professionals remind us that even the research from the field of public relations should have more precise methodological research and should provide not only the answers to solve practical problems (media relations, media trainings, techniques and tools of PR, ...).

For these reasons, it is necessary in this period when the importance of PR is growing (the number of PR workers is increasing, we may talk about so called marketing communicative mix), to deal with this practical discipline also from the academic perspective.

The research may be an inspiration and background for further - more detailed-conceived researches, for the character of this kind of researches requires higher personal and financial provisions.

PR and journalism are in a very interesting and equal relationship of their interdependence and competition and PR is and will be a meaningful, but a specific source. Professional level of a journalist remains his/her essential attribute, as well as his/her ability to distinguish credible and objective information [2].

\section{References}

[1] KOLLARIK, T., SOLLAROVA, E.: Methods of Social-psychological Practice, Bratislava: Ikar, p. 264, ISBN 80-551-0756-3.

[2] PITONAKOVA, S.: Dynamics of Media Space in Slovakia, Dissertation thesis, Nitra: UKF, 2011.

[3] L'ETANG, J.: Public Relations - Basic Theory, Practice, Critical Approach, Praha: Portal, 2009, p. 338, ISBN 978-80-7367-596-7. 